\title{
A Retinex-based Enhancement method for Ocean Remote Sensing Image
}

\author{
Tongyu $\mathrm{Li}^{1}$, Jie Chen ${ }^{2, *}$, Pu Cheng ${ }^{2}$, and $\mathrm{Lu} \mathrm{Yu}^{2}$ \\ ${ }^{1}$ CSSC Ocean Exploration Technology Institute Co.,Ltd, 214135 Wuxi, China \\ ${ }^{2}$ PLA Naval Submarine Academy, 266199 Qingdao, China
}

\begin{abstract}
In order to effectively monitor important sea areas, one of the key issues is the detection of small dynamic targets such as ships. Besides monitoring the hull itself, the monitoring of small targets can also be achieved by detecting ship wakes, which means the sea image with clear texture feature is required. This paper firstly reviews the multiscale retinex (MSR) method, commonly used to enhance the image contrast. Then, it proposes a novel contrast enhancement algorithm base on the subband-decomposed multiscale retinex (SDMSR) method. Experimental results show that our proposed method can make a remarkable enhancement effect for ocean remote sensing images with clouds and whitecap, etc.
\end{abstract}

\section{Introduction}

Since there are abundant material resources in the ocean, how to develop, utilize and protect ocean resources has been a hot issue in many countries. China is a country with vast ocean territory, however, our understanding of the ocean is still very insufficient. On the other hand, we are faced with extremely complex regional disputes, especially in the South China Sea. Therefore, it is crucial to solve the problems of how to effectively monitor important sea areas such as disputed sea areas and route sea areas, ensure the safety of sea traffic and transportation, and maintain the integrity of ocean territory. One of the key issues is the detection of small dynamic targets such as ships.

In addition to monitoring the hull itself, the monitoring of small targets can also be achieved by detecting ship wakes, which means the sea image with clear texture feature is required. Because of high spatial resolution, visible light remote sensing method is an important way to monitor small targets in the sea. The image processing is necessary since visible light remote sensing image can be easily influenced by the factors such as clouds, rain, fog, and light.

At present, image enhancement method can be divided into two types: the frequency domain enhancement and the spatial domain enhancement. In recent years, researchers pay much attention to the image enhancement method based on retinex, which is named for an abbreviation of retina and cortex. As an image enhancement algorithm based on the human visual system, the retinex method performs well in edge enhancement, color fidelity, and dynamic range compression, and can achieve adaptive enhancement for multiple types of images.

The retinex method was originally proposed by Land
$[1,2]$. Then Jobson et al. evolved Land's method to singlescale retinex (SSR) method [3] and multiscale retinex (MSR) method $[4,5]$. Especially, the MSR scheme is effective in dynamic range compression and local contrast enhancement. Then there have been several trials to improve the MSR method in the aspects of edge sharpness [6], halo effects [7], and color correction [8, 9]. Taking the sub-band characteristics into consideration, Jang et al. proposed subband-decomposed multiscale retinex (SDMSR) method $[10,11]$. Experimental results showed that the proposed algorithm can effectively distinguish the details in both highlights and shadows and greatly enhance the overall contrast. We applied the SDMSR method to ocean remote sensing image enhancement, and the image enhancement effect is remarkable in most cases. However, the image enhancement effect is not ideal while the ocean remote sensing image contains clouds, whitecap, etc.

In this paper, we propose a modified SDMSR method which can effectively enhance the ocean remote sensing image with clouds and whitecap. This paper is organized as follows. In section 2, we briefly review the MSR method, which is the basis of the SDMSR method, and our improved algorithm based on the SDMSR method. In section 3, experimental results are presented for several ocean remote sensing images. Finally, the conclusion of this paper is in section 4 .

\section{Materials and Methods}

\subsection{Multiscale Retinex}

As an extended SSR method with multiple kernel windows of different sizes, the output of MSR method is a weighted sum of several different outputs of SSR method [4]. The MSR output is represented as

*Corresponding author: grass2009@163.com 


$$
\log (R(x, y))=\sum_{n=1}^{N} \omega_{n}\left(\log I(x, y)-\log \left(I(x, y) * G_{n}(x, y)\right)\right)
$$

where $N$ denotes the scale number, $I(x, y)$ represents the input image, and $G_{n}(x, y)$ denotes a surround function. The symbol "*" denotes the convolution operation, and $\omega_{n}$ denotes the weight. If $N=3$, then $\omega_{n}=1 / 3 \quad(n=1,2,3)$. The surround function is denoted as following

$$
G_{n}(x, y)=k e^{\frac{-\left(x^{2}+y^{2}\right)}{\sigma_{n}^{2}}}
$$

where $\sigma_{n}$ is a Gaussian surround space constant and $k$ is determined, so that $G_{n}(x, y)$ satisfies the following condition

$$
\iint G_{n}(x, y) d x d y=1
$$

\subsection{Proposed Algorithm}

Based on the MSR method, the subband-decomposed multiscale retinex (SDMSR) method is proposed. The SDMSR method utilizes the subband characteristics of retinex outputs in the aspects of a hybrid intensity transfer function (HITF), subband decomposition, space-varying subband gain, and automatic gain control $[12,13]$. Based on the SDMSR method, we introduce a modified SDMSR method, the processing steps are as follows

The first step is dynamic range compression. To enhance details in both highlights and shadows, the SDMSR method applied a combination of two complementary logarithmic functions, which is called hybrid intensity transfer function (HITF), to an input image. The HITF is represented as following

$$
\begin{gathered}
h_{-} \log (I(x, y))=\omega \cdot \bar{I}(x, y)+(1-\omega) \cdot \tilde{I}(x, y) \\
\bar{I}(x, y)=\log (I(x, y)+1) \\
\tilde{I}(x, y)=\log L-\log (L-I(x, y))
\end{gathered}
$$

where $L$ denotes the image dynamic range (e.g., $L$ is 65536 for a 16-bit image), $I(x, y)$ represents the input image, and $\omega$ denotes weighting parameter of contrast enhancement, which is produced as

$$
\omega=\frac{1}{M} \sum_{(x, y) \in \Omega} \frac{\bar{\zeta}(x, y)}{\bar{\zeta}(x, y)+\tilde{\zeta}(x, y)},
$$

where $\Omega$ denotes a set of all pixel positions, $M$ is the total number of image pixels. $\bar{\zeta}(x, y)$ and $\tilde{\zeta}(x, y)$ represent the local standard deviations (LSDs) at the position $(x, y)$ of $\bar{I}(x, y)$ and $\tilde{I}(x, y)$, respectively. A $5 \times 5$ window is applied to obtain the local statistics.

In the second step, the retinex output $R_{n}(x, y)$ is decomposed into nearly no overlapping spectral bands. Based on the assumption that $R_{n-1}<R_{n}$, the subband decomposed retinex output $\bar{R}_{n}(x, y)$ is calculated as following

$$
\bar{R}_{n}=\left\{\begin{array}{cc}
R_{1}, & n=1 \\
R_{n}-R_{n-1}, & 2 \leq n \leq N
\end{array}\right.
$$

where $N$ denotes the scale number, and the retinex output $R_{n}(x, y), n=1,2, \ldots, N$, is represented as

$$
R_{n}=h_{-} \log (I(x, y))-h_{-} \log \left(G_{n}(x, y) * I(x, y)\right),
$$

where "*" represents the convolution operator, and $G_{n}(x$, $y$ ) is the nth scale surround function, which is defined as following

$$
G_{n}(x, y)=k e^{-\left(x^{2}+y^{2}\right) / \sigma_{n}^{2}}
$$

where $\sigma_{n}$ is a Gaussian surround space constant and $\sigma_{n+1}>\sigma_{n}, n=1,2, \ldots, N-1$. $\mathrm{k}$ is determined so that $G_{n}(x$, y) satisfies

$$
\iint G_{n}(x, y) d x d y=1
$$

The third step is space varying subband gain. The enhanced subband decomposed retinex output $\tilde{R}_{n}$ in the original SDMSR method is defined as

$$
\tilde{R}_{n}=P_{n}(x, y) \cdot \bar{R}_{n}(x, y),
$$

where $P_{n}(x, y)$, which denotes the subband gain function, is defined as

$$
P_{n}(x, y)=\left(\frac{1}{\hat{R}_{n}(x, y)+b}\right)^{1-\hat{\sigma}_{n}},
$$

where

$$
\begin{array}{r}
\hat{R}_{n}(x, y)=\frac{\left|\bar{R}_{n}(x, y)\right|}{\left|\bar{R}_{n}\right|_{\max }}, \\
\hat{\sigma}_{n}=\frac{\sigma_{n}}{\sigma_{\max }},
\end{array}
$$

where

$$
\begin{array}{r}
\left|\bar{R}_{n}\right|_{\max }=\max _{(x, y) \in \Omega}\left\{\left|\bar{R}_{n}(x, y)\right|\right\}, \\
\sigma_{\max }=\max _{n \in\{1,2, \ldots, \mathrm{N}\}}\left\{\sigma_{n}\right\}
\end{array}
$$

In equation $(12), \hat{R}_{n}(x, y)$ represents the normalized subband decomposed retinex output, $\hat{\sigma}_{n}$ represents the normalized Gaussian surround space constant. Constant $b$ in equation (11) is set to 0.2 in the experiment.

In our modified SDMSR method, the normalized subband decomposed retinex output $\hat{R}_{n}(x, y)$ in equation (12) is revised as following

$$
\hat{R}_{n}(x, y)=\frac{\left|\bar{R}_{n}(x, y)\right|}{F_{\bar{R}_{n}}^{-1}(c)}
$$

where constant $\mathrm{c}$ denotes the cumulative probability of $\bar{R}_{n}(x, y)$, and is set to 0.97 in our experiment. $F_{\bar{R}_{n}}^{-1}(c)$ represents the inverse function of the cumulative probability function of $\bar{R}_{n}(x, y)$.

Then enhance the details of high-frequency subband by using the detail adjustment function. The enhanced output $\hat{I}(x, y)$ of the SDMSR method is given by

$$
\hat{I}(x, y)=D A F(x, y) \cdot \tilde{R}_{1}(x, y)+\sum_{n=2}^{N} \tilde{R}_{n}(x, y)
$$

where $D A F(x, y)$ is the detail adjustment function and is defined as

$$
D A F(x, y)=1-\lambda \cdot(\hat{\zeta}(x, y)-0.5)^{2}, \quad 0 \leq \lambda \leq 4,
$$


where $\hat{\varsigma}(x, y)$ represents the normalized LSD, which is calculated as following

$$
\hat{\zeta}(x, y)=\frac{\varsigma(x, y)}{\varsigma_{\max }}, \text { where } \varsigma_{\max }=\max _{(x, y) \in \Omega}\{\varsigma(x, y)\}
$$

where $\varsigma(x, y)$ represents the LSD at pixel position $(x, y)$ of the input image $I(x, y)$, and is obtained by using a $5 \times 5$ window.

In the last step, for the final enhancement to display, the automatic gain control is needed to transform the output $\hat{I}(x, y)$ of the SDMSR method from the logarithmic domain to the display domain. The automatic gain control in the original SDMSR method is defined as

$$
I_{\text {out }}(x, y)=\frac{L-1}{\hat{I}_{\max }-\hat{I}_{\min }} \cdot\left[\hat{I}(x, y)-\hat{I}_{\min }\right] \text {, }
$$

where $I_{\text {out }}(x, y)$ represents the final enhanced image, $\hat{I}_{\min }=\min _{(x, y) \in \Omega}\{\hat{I}(x, y)\}$, and $\hat{I}_{\max }=\max _{(x, y) \in \Omega}\{\hat{I}(x, y)\}$.

In our modified SDMSR method, the automatic gain control in equation (20) is revised as

$$
I_{\text {out }}(x, y)=\frac{L-1}{F_{\hat{I}}^{-1}(c)-\hat{I}_{\min }} \cdot\left[\hat{I}(x, y)-\hat{I}_{\min }\right]
$$

where $\hat{I}_{\min }=\min _{(x, y) \in \Omega}\{\hat{I}(x, y)\}$, constant $c$ denotes the cumulative probability of $\hat{I}(x, y)$, and is set to 0.97 in our experiment. $F_{\hat{I}}^{-1}(c)$ represents the inverse function of the cumulative probability function of $\hat{I}(x, y)$.

\section{Results}

To evaluate the proposed method, we adopted two 16-bit ocean remote sensing image with clouds and whitecap, and compared our algorithm with SSR method, MSR method, and original SDMSR method. The experimental results are shown in fig. 2 . In this experiments, the scale factor of Gaussian surround function in the SSR method is $\sigma=100$, and in other methods is $\sigma=30,100,300$. The convolution kernel size of Gaussian surround function in all methods is $200 \times 200$.

As shown in fig. 1, the contrast in the original image is weak. By applying the SSR method, the MSR method, and the original SDMSR method to the remote sensing image, respectively, the contrast of the image has been improved to a certain extent. However, the texture of the enhanced images is still not clear enough. Compared with the other three methods, our method highlights the details of the ocean remote sensing image better.

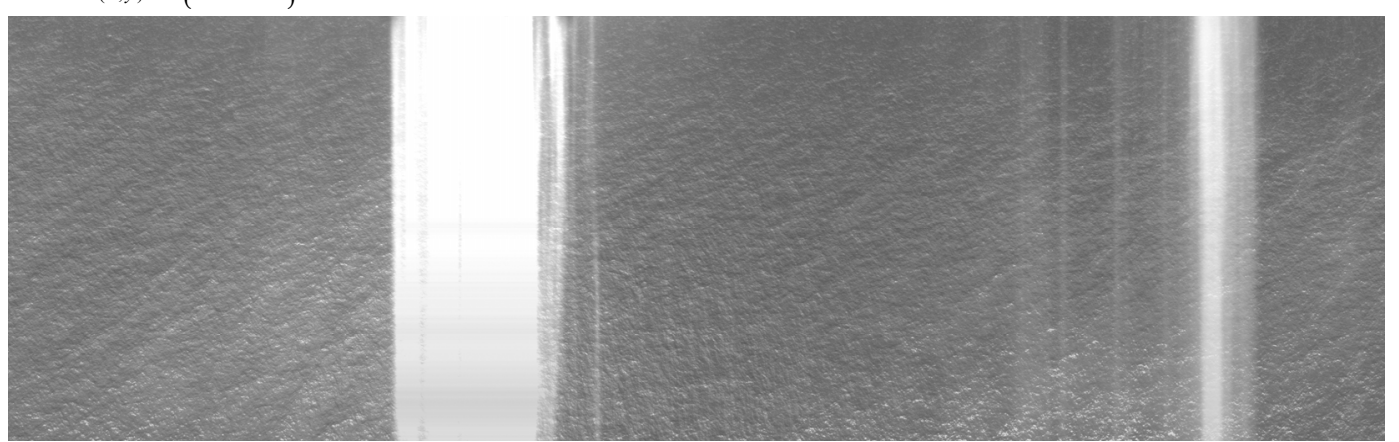

(a) original image

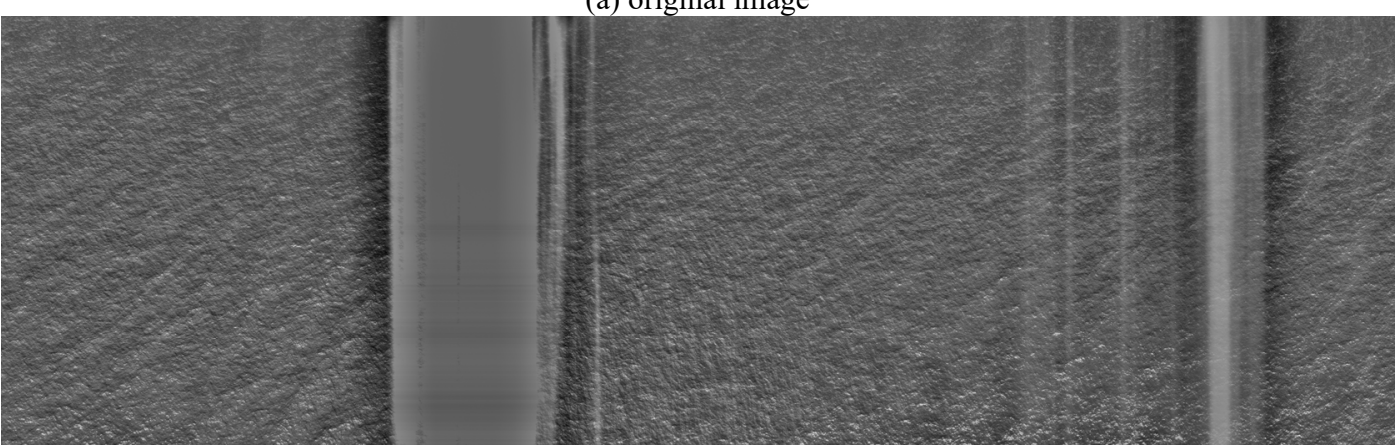

(b) result of the SSR method

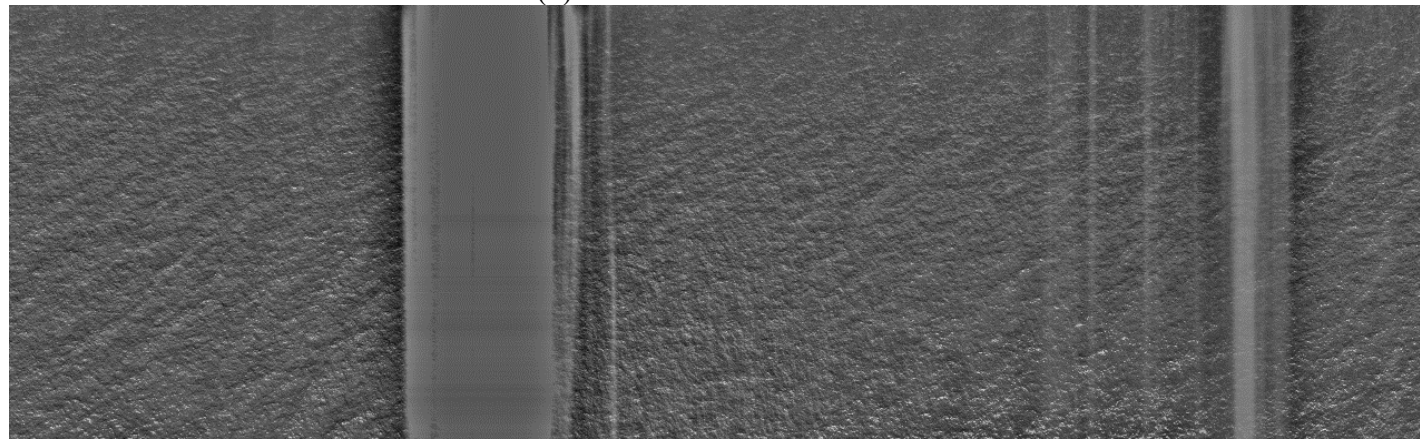

(c) result of the MSR method 

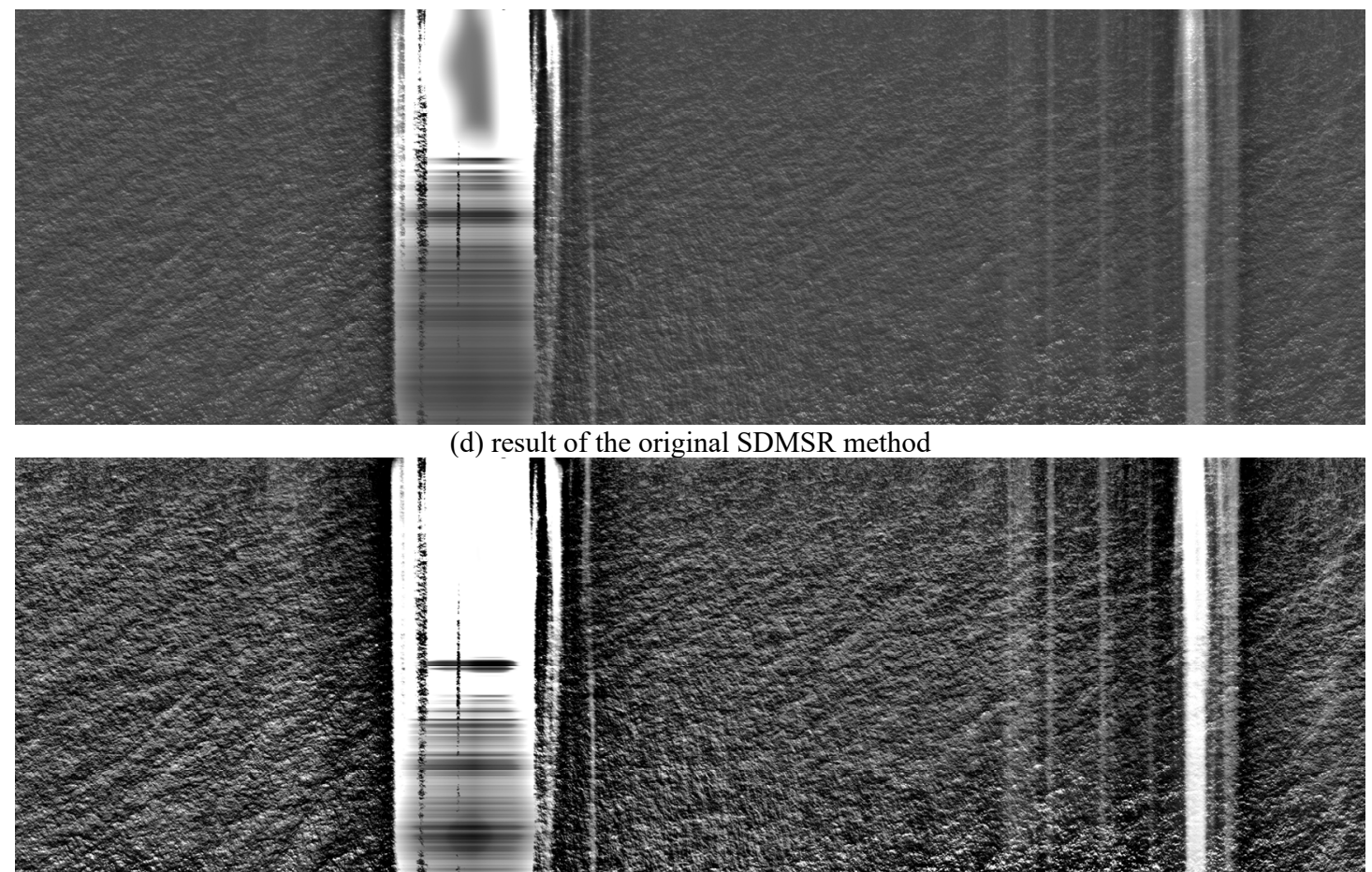

(e) result of the proposed method

Fig. 1. The results of ocean remote sensing image enhanced by different methods

\section{Conclusions}

In order to effectively monitor important sea areas, the sea image with clear texture feature is required. However, the image enhancement effect based on the SDMSR method is not ideal while the ocean remote sensing image contains clouds, whitecap, etc. Therefore, in this paper, we modified the original SDMSR method by revising the subband gain function and the automatic gain control function. Experiment results show that our proposed method can effectively enhance the clarity of the ocean remote sensing image with clouds and whitecap.

\section{References}

1. E. H. Land, and J. J. McCann. Lightness and retinex theory. J. Opt. Soc. Am, 61: 1-11 (1971).

2. E. H. Land. An alternative technique for the computation of the designator in the retinex theory of color vision. Proc. Nat. Acad. Sci., 83: 3078-3080 (1986).

3. D. J. Jobson, Z. Rahman, and G. A. Woodell. Properties and performance of a center/surround retinex. IEEE Trans. Image Processing, 6: 451-462 (1997).

4. D. J. Jobson, Z. Rahman, and G. A. Woodell. A multiscale retinex for bridging the gap between color images and the human observation of scenes. IEEE Trans. Image Processing, 6: 965-976 (1997).

5. Z. Rahman, D. J. Jobson, and G. A. Woodell. Retinex processing for automatic image enhancement. $J$. Electronic Imaging, 13: 100-110 (2004).

6. G. Orsini, G. Ramponi, P. Carrai, and R. Di Federico. A modified retinex for image contrast enhancement and dynamics control. In: Int. Conf. Image Process., Barcelona. pp. 393-396 (2003).

7. B. Sun, W. Chen, H. Li, W. Tao, and J. Li. Modified luminance based adaptive MSR. In: IEEE ICIG, Chengdu. pp. 116-120 (2007).

8. K. Barnard and B. Funt. Analysis and improvement of multiscale retinex. In: Fifth Color Imaging Conference: Color Science, Systems and Applications, Scottsdale. pp. 221- 226 (1997).

9. T. Watanabe, Y. Kuwahara, A. Kojima, and T. Kurosawa. Improvement of color quality with modified linear multi-scale retinex. In: 15th SPIE Symposium on Electronic Imaging, Santa Clara. pp. 59-69 (2003).

10. J. H. Jang, Y. Bae, and J. B. Ra. Multi-sensor image fusion using subband decomposed multiscale retinex. In: 16th IEEE Int. Conf. Image Process., Cairo. pp. 2177-2180 (2009).

11. J. H. Jang, Y. Bae, and J. B. Ra. Contrast-Enhanced Fusion of Multisensor Images Using SubbandDecomposed Multiscale Retinex. IEEE Transactions on Image Processing, 21: 3479-3490 (2012).

12. J. H. Jang, S. D. Kim, and J. B. Ra. Enhancement of optical remote sensing images by subbanddecomposed multiscale retinex with hybrid intensity transfer function. IEEE Geosci. Remote Sensing Lett., 8: 983-987 (2011).

13. J. H. JANG, B. CHOI, S. D. Kim, et al. Sub-band decomposed multiscale retinex with space varying gain. In: IEEE Int. Conf. Image Process., San Diego. pp. 3168-3171 (2008). 